\title{
Vedolizumab in patients with concurrent primary sclerosing cholangitis and inflammatory bowel disease does not improve liver biochemistry but is safe and effective for the bowel disease
}
B. Christensen ${ }^{1,2,3}$
D. Micic $^{4}$
P. R. Gibson ${ }^{1}$
A. Yarur ${ }^{5}$
E. Bellaguarda ${ }^{4}$
P. Corsello ${ }^{6}$
J. N. Gaetano ${ }^{3}$
J. Kinnucan ${ }^{6}$
V. L. Rao ${ }^{3}$
S. Reddy ${ }^{4}$
S. Singh ${ }^{5}$
J. Pekow ${ }^{3}$ | D. T. Rubin ${ }^{3}$

${ }^{1}$ Alfred Hospital and Monash University, Melbourne, Australia

${ }^{2}$ Royal Melbourne Hospital, Melbourne, Australia

${ }^{3}$ University of Chicago Medicine Inflammatory Bowel Disease Center, Chicago, IL, USA

${ }^{4}$ Northwestern University Feinberg School of Medicine, Chicago, IL, USA

${ }^{5}$ Division of Gastroenterology and Hepatology, Medical College of Wisconsin, Milwaukee, WI, USA

${ }^{6}$ University of Michigan Health System, Ann Arbor, MI, USA

Correspondence

Dr. B Christensen, Gastroenterology

Department, The Royal Melbourne Hospital,

Parkville, Victoria, Australia.

Email: britt.christensen@mh.org.au

Funding information

NIH, Grant/Award Number: P30DK42086, K08DK090152

\section{Summary}

Background: Blocking of lymphocyte trafficking to bile ducts is a potential mechanism to alter the disease course of patients with primary sclerosing cholangitis (PSC).

Aim: To describe the effect of the $\alpha_{4} \beta_{7}$ integrin antibody, vedolizumab, on liver biochemistry and disease activity in patients with PSC and inflammatory bowel disease (IBD).

Methods: This is a retrospective multi-centre study of adult patients with a diagnosis of both IBD and PSC. The primary outcome was change in serum alkaline phosphatase level at weeks 14 and 30. Secondary outcomes included changes in other liver biochemistries and in clinical outcomes for the bowel disease. A safety analysis for adverse events was performed.

Results: Thirty-four patients (16 Crohn's disease, 18 ulcerative colitis) were included. Nine (26\%) had a history of liver transplant. Median follow-up on vedolizumab was 9 months (IQR: 7-16). There was no overall change in serum alkaline phosphatase level with vedolizumab therapy (median 268 [IQR: 105-551] IU/L at baseline versus 249 [IQR: 183-634] IU/L, $P=0.99$ at week 30). No significant changes in other liver biochemistries or the Mayo PSC Risk Score were demonstrated at week 30. Clinical remission was achieved at week 30 in 55\% of Crohn's disease and $29 \%$ of ulcerative colitis patients. Seven (21\%) patients ceased vedolizumab; six patients stopped therapy due to persistent IBD activity and one for worsening of liver biochemistries.

Conclusion: Vedolizumab treatment in patients with PSC and IBD did not improve liver biochemistry but was associated with improvement in bowel disease and a favourable safety profile. 


\section{1 | INTRODUCTION}

Primary sclerosing cholangitis (PSC) causes chronic and progressive injury to the bile ducts characterised by inflammatory and obliterative periductal fibrosis, and is the classic hepatobiliary extra-intestinal manifestation of inflammatory bowel disease (IBD). ${ }^{1}$ With disease progression, progressive biliary strictures can lead to cholangitis, biliary cirrhosis and end-stage liver disease. ${ }^{1}$ Two-thirds of cases of PSC cases are associated with IBD ${ }^{2}$ and, although patients are commonly asymptomatic at the time of diagnosis, they have a shorter than average survival compared to matched controls in the general population. ${ }^{3,4}$

As PSC is associated with significant morbidity and mortality, various therapies have been examined in an effort to mitigate the progressive nature of the disease. Immunosuppressive agents including corticosteroids, tacrolimus, ciclosporin, azathioprine, methotrexate and anti-tumour necrosis factor (TNF) therapies have not shown clinical benefit in PSC. ${ }^{3}$ Ursodeoxycholic acid, a hydrophilic bile acid that is often employed to treat cholestatic liver diseases, ${ }^{3,5}$ has demonstrated improvement in alkaline phosphatase and other liver biochemistry in patients with PSC but has not favourably influenced key endpoints that include death, liver transplantation or progression to cirrhosis. $1,3,6,7$

Vedolizumab is a selective humanised monoclonal antibody to the $\alpha_{4} \beta_{7}$ integrin expressed on lymphocytes. The binding of the $\alpha_{4} \beta_{7}$ integrin to MadCAM-1, which is expressed on intestinal endothelial vessels, allows for gut lymphocyte trafficking. ${ }^{8}$ Thus vedolizumab modulates the ability of lymphocytes to enter the gastrointestinal epithelium, reducing inflammation and inducing mucosal healing in patients with moderate-severe Crohn's disease (CD) and ulcerative colitis (UC). ${ }^{9-11}$ Although MAdCAM-1 is not expressed in normal liver tissue, it is induced in the portal tract endothelium of inflamed and cirrhotic livers, and its activity correlates with histological inflammatory activity in PSC. ${ }^{12,13}$ It has therefore been postulated that vedolizumab could also be of therapeutic benefit in patients with PSC. However, experience with vedolizumab in patients with PSC has been limited to individual-center case series. $^{14,15}$

We studied the use of vedolizumab in a multi-centre, multinational cohort of patients with PSC and IBD with a primary focus on change in liver biochemistry. Secondary outcomes assessed for changes in prognostic models of PSC and clinical outcomes and safety of vedolizumab in patients with chronic liver disease and IBD including patients with orthotopic liver transplant.

\section{2 | MATERIALS AND METHODS}

\section{1 | Participants}

Electronic medical records at participating sites were reviewed for adult patients with an established diagnosis of concurrent IBD and PSC (IBD-PSC) based on clinical, biochemical, imaging and endoscopic information and who had been initiated on vedolizumab between June 2014 and January 2016. Data were collected until August 2016. Participating sites included: University of Chicago Medicine $(n=11)$, Medical College of Wisconsin $(n=9)$, University of Michigan ( $n=7)$, Northwestern University $(n=4)$ and Alfred Hospital, Melbourne, Australia $(n=3)$. These sites were identified by a pre-existing collaborative group without prior knowledge to the number of patients that would meet the inclusion criteria. All patients that met the inclusion criteria from each site were included in the study. Institutional review board approval was granted at the individual participating sites.

\section{2 | Study design}

A retrospective cohort study was performed. Baseline demographic information abstracted from the medical record included age, sex, dates of diagnosis, disease phenotype based on the Montreal classification, ${ }^{16}$ and previous and current use of ursodeoxycholic acid, antiinflammatory agents and/or immunosuppressant therapy (steroids, immunomodulators, anti-TNF agents). Changes to immunomodulator therapy and UDCA dosing were monitored throughout the study. Results of orthotopic liver transplant, endoscopic retrograde cholangiopancreatography (ERCP), magnetic resonance cholangiopancreatography (MRCP) and liver biopsy before and during vedolizumab treatment were recorded. Clinical scores, laboratory values and endoscopic outcomes were collected from standard-of-care visits. In addition, all adverse events including hospitalisations, surgeries, infusion reactions or infections after initiation of vedolizumab were documented.

\section{3 | Outcomes}

The primary outcome of interest was a decrease in alkaline phosphatase level at weeks 14 and 30 in those with active PSC (patients with PSC who had not undergone orthotopic liver transplant and those who underwent orthotopic liver transplant with recurrent PSC in the transplanted liver). Secondary outcomes of interest included changes in total bilirubin, Mayo PSC Risk Score, ${ }^{17}$ alanine aminotransferase and aspartate aminotransferase at weeks 14 and 30 from baseline in those with active PSC, and the development of adverse events at any time. Adverse events were defined as any clinically significant event that occurred from the date of commencing vedolizumab to the last follow-up. Adverse events were graded as serious if they resulted in discontinuation of vedolizumab, hospitalisation or death.

Clinical activity was assessed using the Harvey-Bradshaw Index (HBI) for $\mathrm{CD}^{18}$ and the Simple Clinical Colitis Activity Index (SCCAI) for UC. ${ }^{19}$ In those with clinical disease activity at baseline, rates of clinical remission and corticosteroid-free remission at week 14 and 30 were determined. Clinical remission was defined as a $\mathrm{HBI} \leq 4^{18}$ or a SCCAI $\leq 2 .{ }^{19}$ Corticosteroid-free remission was defined as clinical remission without need for concomitant corticosteroids.

In patients with baseline endoscopy and follow-up colonoscopy after at least 3 months of vedolizumab, endoscopic response was 
assessed utilising the SES-CD for CD patients ${ }^{20}$ or Mayo endoscopic subscore for UC. ${ }^{21}$ In $C D$, endoscopic improvement was defined as reduction in the SES-CD $>50 \%$ and mucosal healing as SES-CD score $<3$. In UC, endoscopic improvement was defined as absolute reduction $\geq 1$ point in the Mayo endoscopic subscore and mucosal healing as Mayo endoscopic subscore of 0 or 1 . Biopsies in $C D$ and UC were scored on a 4-point scale as quiescent/normal (0), mild (1), moderate (2) or severe (3). ${ }^{22}$ Histological improvement was defined as an absolute reduction of 1 point or more and histological remission as score of 0 .

\subsection{Statistical methods}

Patients were analysed on an intent-to-treat basis and cessation of vedolizumab for any reason was considered treatment failure. Descriptive statistics were provided to summarise demographic characteristics using mean (95\% confidence interval [CI]) or median (interquartile range [IQR]) for continuous variables, and number and percentage for categorical variables. As the differences between liver biochemistry were not normally distributed, the Wilcoxon's signed-rank test was used for statistical analysis of response to treatment. Pre-treatment and post-treatment biochemical indices and Mayo PSC Risk Scores were compared between week 0 and week 14 and week 0 and week 30 . For patients who withdrew prematurely, the last observation was carried forward. A two-sided Pvalue $\leq 0.05$ was considered statistically significant. All data analyses were performed using Stata 12.0 (STATACORP, College Station, TX, USA).

\section{3 | RESULTS}

\section{1 | Baseline characteristics}

Demographics, baseline characteristics and medication usage of the 34 patients with PSC-IBD who met inclusion criteria are shown in Table 1. Included patients and clinical outcomes assessed are outlined in Figure 1 . Of the nine $(26 \%)$ patients who had undergone orthotopic liver transplantation for PSC prior to initiation of vedolizumab, 3 had recurrent PSC demonstrated on liver biopsy. Thus, 28 patients (71\% large duct) had active PSC at the time of treatment with vedolizumab.

Vedolizumab was commenced for IBD clinical disease activity in the majority of patients ( $n=27,79 \%$ ). Other indications for vedolizumab included possible therapeutic benefit in active PSC $(n=3)$, intolerance of previous maintenance medication $(n=1)$, transition from natalizumab $(n=1)$ and severe endoscopic disease activity despite clinical remission $(n=1)$. Median clinical follow-up while on vedolizumab was 9 (IQR: 7-16) months and 28 (82\%) patients had at least 6 months of clinical follow-up.

At commencement of vedolizumab, 7 patients were on long-term ursodeoxycholic acid, the dose of which did not change in these patients throughout the study period. Two patients commenced ursodeoxycholic acid during the study period.

\subsection{Efficacy}

\subsubsection{Alkaline phosphatase}

Alkaline phosphatase levels from all patients with active PSC and biochemical testing before and after vedolizumab are shown in Table 2 and Figure 2. Overall, there was no significant change in alkaline phosphatase levels before and after treatment with vedolizumab at week 14 or 30. Median alkaline phosphatase activities were 268 (IQR: 105551) IU/L before treatment, 234 (IQR: 126-396) IU/L, $P=0.346$ at week 14 and 249 (IQR: 183-634) IU/L, $P=0.990$ at week 30. The median percentage change from baseline in alkaline phosphatase was 0\% [IQR: $-17 \%, 10 \%]$ at week 14 and $-1 \%$ [IQR: $-20 \%, 21.7 \%$ ] at week 30 .

Of the 18 patients (69\%) with an elevated alkaline phosphatase at baseline, 11 patients (61\%) improved but none achieved a normal alkaline phosphatase at week 30 (Figure 2A). Alkaline phosphatase did significantly fall with treatment at week 14 from median 475 (IQR: 241-757) IU/L at baseline to 322.5 (IQR: 220-651) IU/L at week $14(P=0.025)$. However, two patients potentially confounded this analysis with a fall associated with the commencement of ursodeoxycholic acid (where alkaline phosphatase activities fell by $75 \%$ and $13 \%$, respectively, as shown in Figure $2 \mathrm{~A}$ ). At week 30 , median alkaline phosphatase activities only trended down to 283 (IQR: 207-658) IU/L $(P=0.267)$. When patients who were commenced on ursodeoxycholic acid during vedolizumab treatment were excluded, the decrease in alkaline phosphatase only trended to significance at week $14(P=0.070)$ and was again not significant at week $30(P=0.866)$. The median percentage change in alkaline phosphatase among individuals with an elevated baseline level was $-10 \%$ [IQR: $-38 \%, 0 \%$ ] at week 14 and $-12 \%$ [IQR: $-24 \%, 2 \%$ ] at week 30. In most cases, improvement was evident by week 14; only one patient with transient worsening of their alkaline phosphatase at week 14 achieved improvement in their alkaline phosphatase at week 30. No clear demographic or clinical characteristics, including the duration of PSC, type of PSC (small-duct vs large-duct) and type of IBD (CD vs UC), defined patients with alkaline phosphatase improvement (data not shown).

Of the eight patients (31\%) with normal alkaline phosphatase at baseline, four (50\%) had a subsequent increase in its activity to abnormal levels over the 30 weeks of treatment (Figure 2B). Overall, in these eight patients, there was a significant increase in alkaline phosphatase from a baseline median of 98 (IQR: 77-102) IU/L to 110 (IQR: 102-183) IU/L, $P=0.019$ at week 14 and to 146 (IQR: 90-203) IU/L, $P=0.036$ at week 30 . The median percentage change among individuals with a normal baseline alkaline phosphatase was $+20 \%$ (IQR: $5 \%$, $80 \%$ ) at week 14 and +48\% (IQR: 4\%, 94\%) at week 30 .

\subsection{2 | Total bilirubin, aspartate aminotransferase, alanine aminotransferase and Mayo PSC Risk Score}

As shown in Table 2 and Figure 3, there were no significant changes in the median serum total bilirubin, aspartate aminotransferase or 
TABLE 1 Baseline characteristics of patients with primary sclerosing cholangitis (PSC) and inflammatory bowel disease (IBD)

\begin{tabular}{|c|c|c|}
\hline Characteristic & Crohn's disease, $n=16$ & Ulcerative colitis, $n=18$ \\
\hline $\begin{array}{l}\text { All patients: } n=34 \\
\text { Male gender, } n(\%)\end{array}$ & $9(56 \%)$ & 15 (83\%) \\
\hline Median age, y (IQR) & $34(25.5-38.5)$ & $37(23-46)$ \\
\hline Median duration of disease, y (IQR) & $10.5(7.5-18.5)$ & $10(3-15)$ \\
\hline Current smoker, $\mathrm{n}(\%)$ & $2(13 \%)$ & $0(0 \%)$ \\
\hline Clinical disease activity at baseline, $\mathrm{n}(\%)$ & $\begin{array}{l}\mathrm{HBI} \\
<5 \text { (remission): } 5(31 \%) \\
\text { 5-7 (mild): } 5(31 \%) \\
\text { 8-16 (moderate): } 6(38 \%) \\
>16 \text { (severe): } 0(0 \%)\end{array}$ & $\begin{array}{l}\text { SCCAI } \\
<3 \text { (remission): } 4(22 \%) \\
\text { 3-6 (mild): } 8 \text { (44\%) } \\
\text { 7-10 (moderate): } 4(22 \%) \\
>10 \text { (severe): } 2(11 \%)\end{array}$ \\
\hline Recurrent PSC in transplanted liver, $\mathrm{n}(\%)$ & $1(50 \%)$ & $2(25 \%)$ \\
\hline Active PSC at vedolizumab commencement, $\mathrm{n}(\%)$ & 15 (94\%) & $13(72 \%)$ \\
\hline Anti-TNF treatment naïve, $\mathrm{n}(\%)$ & $1(6 \%)$ & $6(33 \%)$ \\
\hline \multicolumn{3}{|l|}{ Concomitant medications at commencement, $\mathrm{n}$ (\%) } \\
\hline Tacrolimus & $2(13 \%)$ & 7 (39\%) \\
\hline Immunomodulator & $6(38 \%)$ & 7 (39\%) \\
\hline Glucocorticoids & $4(25 \%)$ & 8 (44\%) \\
\hline Antibiotics & $1(6 \%)$ & $1(6 \%)$ \\
\hline Median prednisolone equivalent dose, mg (IQR) & $40(30-40)$ & $15(10-40)$ \\
\hline On UDCA, n (\%) & $5(36 \%)$ & $2(17 \%)$ \\
\hline Median daily urosodeoxycholic acid dose, mg (IQR) & $900(900-1000)$ & $1000(1000-1000)$ \\
\hline \multicolumn{3}{|l|}{ Biochemistry at baseline, median (IQR) } \\
\hline Alkaline phosphatase (IU/L) (normal <120) & $268(99-551)$ & $283(108-618)$ \\
\hline Aspartate aminotransferase (IU/L) (normal <30) & $34(24-98)$ & $81(50-111)$ \\
\hline Alanine aminotransferase (IU/L) (normal <120) & $42(20-144)$ & $86(27-139)$ \\
\hline Albumin (g/dL) (normal 3.9-4.4) & $3.5(2.9-4.5)$ & $4.1(3.9-4.3)$ \\
\hline Total bilirubin (mg/dl) & $0.5(0.4-0.6)$ & $0.8(0.6-1.6)$ \\
\hline Baseline Mayo Risk Score, mean (95\% Cl) & $-0.55(-1.38-0.27)$ & $-0.26(-0.81-0.29)$ \\
\hline
\end{tabular}

alanine aminotransferase over 14 or 30 weeks' therapy with vedolizumab. The calculated Mayo PSC Risk Score for PSC did improve significantly from baseline to week 14 from mean $-0.40[95 \% \mathrm{Cl}:-0.85$, 0.05] at baseline to $-0.59[95 \% \mathrm{Cl}:-0.99,-0.18]$ at week 14 $(P=0.03)$. This difference was no longer significant at week 30 with a Mayo PSC Risk Score of -0.38 [95\% Cl: $-0.83,0.08$ ] ( $P=0.90)$ (Figure 3D).

\subsection{Clinical activity of intestinal disease}

All 34 patients had clinical assessment of their intestinal disease activity before and after vedolizumab therapy was initiated and 25 patients (11 CD; $14 \mathrm{UC}$ ) had clinically active IBD (HBI > 4 or SCCAI > 2) at baseline. Among those with CD, 5 (45\%) patients achieved clinical remission by week 14 , increasing to $6(55 \%)$ by week 30 . In those with 
34 Patients with a diagnosis of PSC and included in study

FIGURE 1 Flow chart of study design and included patients

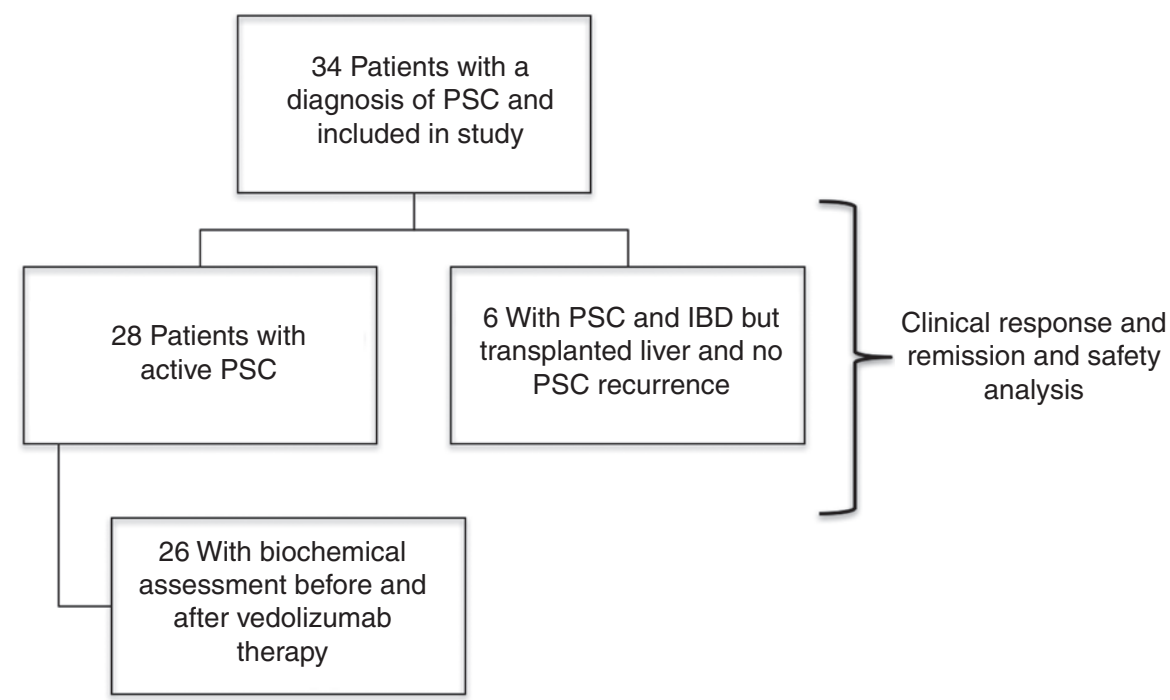

TABLE 2 Change in liver biochemistry and Risk Score with vedolizumab

\begin{tabular}{|c|c|c|c|c|c|}
\hline & Baseline & Wk 14 & $\begin{array}{l}\text { P-value: difference } \\
\text { from baseline }\end{array}$ & Wk 30 & $\begin{array}{l}\text { P-value: difference } \\
\text { from baseline }\end{array}$ \\
\hline $\begin{array}{l}\text { Alkaline phosphatase (IU/L) } \\
\text { median (IQR) }\end{array}$ & $268(105,551)$ & $265(176,508)$ & 0.346 & $236(183,634)$ & 0.990 \\
\hline $\begin{array}{c}\text { Bilirubin (IU/L) } \\
\text { median (IQR) }\end{array}$ & $0.6(0.4,0.9)$ & $0.7(0.4,1)$ & 0.619 & $0.7(0.4,1.3)$ & 0.960 \\
\hline $\begin{array}{l}\text { AST (IU/L) } \\
\text { median (IQR) }\end{array}$ & $54(27,98)$ & $37(23,75)$ & 0.215 & $46(39,93)$ & 0.693 \\
\hline $\begin{array}{l}\mathrm{ALT}(\mathrm{IU} / \mathrm{L}) \\
\text { median (IQR) }\end{array}$ & $63(20,144)$ & $50(31,107)$ & 0.459 & $58(39,154)$ & 0.809 \\
\hline $\begin{array}{l}\text { Mayo PSC Risk Score } \\
\text { Mean }(95 \% \mathrm{Cl})\end{array}$ & $-0.40(-0.85-0.05)$ & $-0.59(-0.99$ to -0.18$)$ & $0.030^{\mathrm{a}}$ & $-0.38(-0.83-0.08)$ & 0.879 \\
\hline
\end{tabular}

${ }^{a}$ Signifies statistical significant difference.

UC, 3 (21\%) achieved clinical remission by week 14, increasing to four (29\%) by week 30 . Of the 12 patients (4CD; $8 U C$ ) who were on corticosteroid therapy at baseline, 10 (83\%) (3CD; 7UC) were weaned from corticosteroids during follow-up and 4 (33\%) (2CD; 2UC) achieved corticosteroid-free remission by week 30 (Figure 4A). Eight of 9 (89\%) patients in clinical remission at initiation of therapy remained in clinical remission through to 30 weeks.

\section{4 | Mucosal healing}

Thirteen patients (6CD; 7UC) had baseline endoscopic disease activity and follow-up assessment for mucosal healing at median time of 6 (IQR: 5, 10) months. Of the 6 CD patients, two (33\%) achieved endoscopic improvement, but none achieved mucosal healing. None of five patients with $C D$ who had histological assessment showed histological improvement or healing. Of the seven UC patients, two (29\%) achieved endoscopic improvement and one (14\%) mucosal healing. Six of those patients had histological assessment; 3 (50\%) achieved histological improvement and 1 (17\%) histological remission (Figure 4B). There was no association between mucosal improvement and change in serum alkaline phosphatase activity, with $33 \%$ and $29 \%$ of those who had deterioration and improvement of their alkaline phosphatase, respectively, achieving endoscopic improvement with vedolizumab treatment $(P=1.00)$.

\subsection{Safety and adverse events}

Median follow-up was 9 (IQR: 7, 16) months. Seven (21\%) patients ceased vedolizumab after a median of 8 (IQR: 5,8$)$ months, six for ongoing clinical disease activity and one for deteriorating LFTs. The patient with worsening LFTs had normal liver biochemistry at baseline; the alkaline phosphatase increased to $351 \mathrm{IU} / \mathrm{L}$ and alanine aminotransferase $264 \mathrm{IU} / \mathrm{L}$ at week 14 . This patient proceeded to liver biopsy with histological findings consistent with a drug reaction thought secondary to vedolizumab. Vedolizumab was ceased at week 16 and the liver biochemistries returned to normal over the following 3 months. Two further patients had liver-related complications and were hospitalised for cholangitis, but continued on vedolizumab. Of these, one was found to have a dominant stricture that was dilated at ERCP, and the other patient proceeded to liver transplantation. One patient was hospitalised for poorly controlled intestinal disease, resulting in colectomy. 


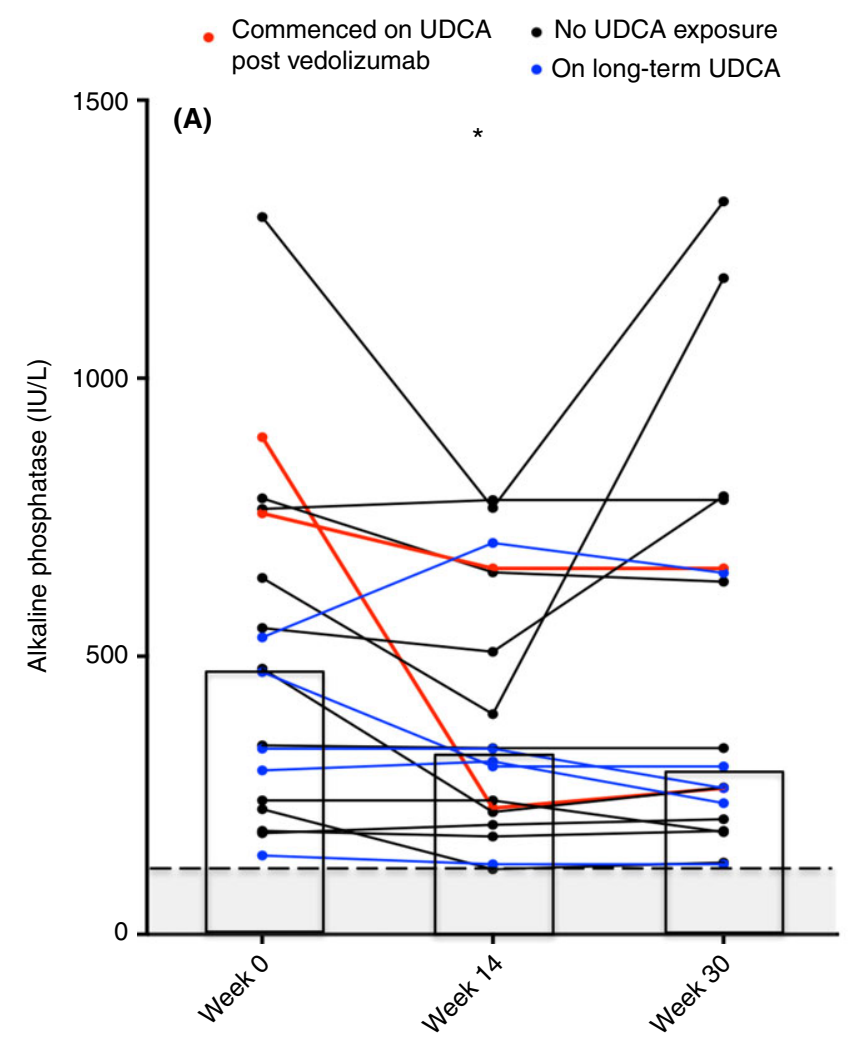

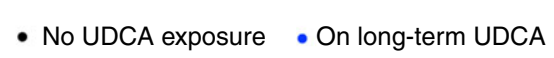

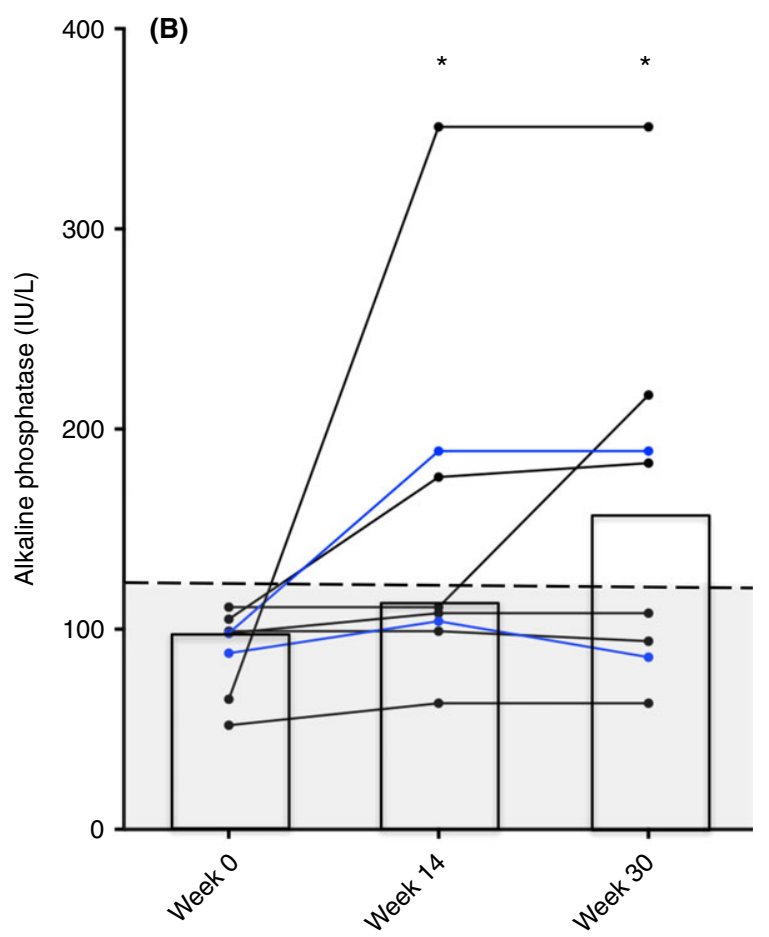

FIGURE 2 Change in Alkaline Phosphatase following treatment with vedolizumab. *Indicates significant decrease $(P<0.05)$ from wk 0 level. Shaded region represents normal range of alkaline phosphatase ( $<120 \mathrm{IU} / \mathrm{L})$. The columns indicate the median value for each group. (A) Patients with elevated alkaline phosphatase ( $\geq 120 \mathrm{IU} / \mathrm{L}$ ) level at baseline. There was a statistically significant decrease at wk $14(P=0.025)$. This decrease was no longer statistically significant at wk $30(P=0.267)$. (B) Patients with normal alkaline phosphatase activities at baseline. There was a significant increase at week $14(P=0.02)$ and $30(P=0.04)$

There were four (12\%) minor adverse events that did not require hospitalisation, change in therapy, or medical intervention. They included one patient who developed an upper respiratory tract infection, one with headaches, one dental abscess and one with diarrhoea associated with Aeromonas on stool culture.

\section{DISCUSSION}

Despite multiple studies investigating treatment options for PSC, currently there is no effective medical therapy. It has been postulated that vedolizumab, a selective $\alpha_{4} \beta_{7}$ integrin antibody, may alter the disease course of progressive PSC by blocking lymphocyte trafficking to bile ducts, which, during chronic inflammation, express MadCAM-1. However, the findings of the current multi-centre, multi-national cohort suggest that vedolizumab has little impact on liver biochemistry or the Mayo PSC Risk Score in the vast majority of patients with PSC. Some patients did demonstrate a small and persistent decrease in the serum alkaline phosphatase following initiation of vedolizumab, but likewise there were several patients who commenced the study with normal alkaline phosphatase levels and also had small subsequent increases in their alkaline phosphatase.

While our findings support a tendency in patients with elevated alkaline phosphatase and PSC-IBD to decrease the enzymes concentration early in follow-up, the effect was not sustained through 30 weeks, nor did it represent a clinically meaningful change in only $10 \%$ difference in alkaline phosphatase following vedolizumab treatment. In addition, four of eight patients with normal alkaline phosphatase at commencement of therapy developed abnormal levels over 30 weeks of therapy and the overall increase in alkaline phosphatase levels in these patients was statistically significant at both week 14 and week 30. Of note, this increase was not due to the PSC in all patients and, despite this increase, only one patient required cessation of vedolizumab secondary to drug-induced liver damage and not progression of their PSC. However, the overall changes in alkaline phosphatase, both up and down, were small and appeared clinical inconsequential. Certainly, the short-term biochemical effects in this study do not inspire confidence that longer term results will be any more impressive.

Whether vedolizumab slows the progression of alkaline phosphatase increase cannot be ascertained without a control group. Reduction in alkaline phosphatase has been associated with longer survival in PSC, and a recent PSC study group consensus statement identified alkaline phosphatase as a potentially promising surrogate endpoint for PSC clinical trials. ${ }^{23}$ However, the potential that changes in liver biochemical profile do not reflect long-term progression of liver disease must be taken into account in interpreting the current results. Lessons from experience with ursodeoxycholic acid, 
(A)

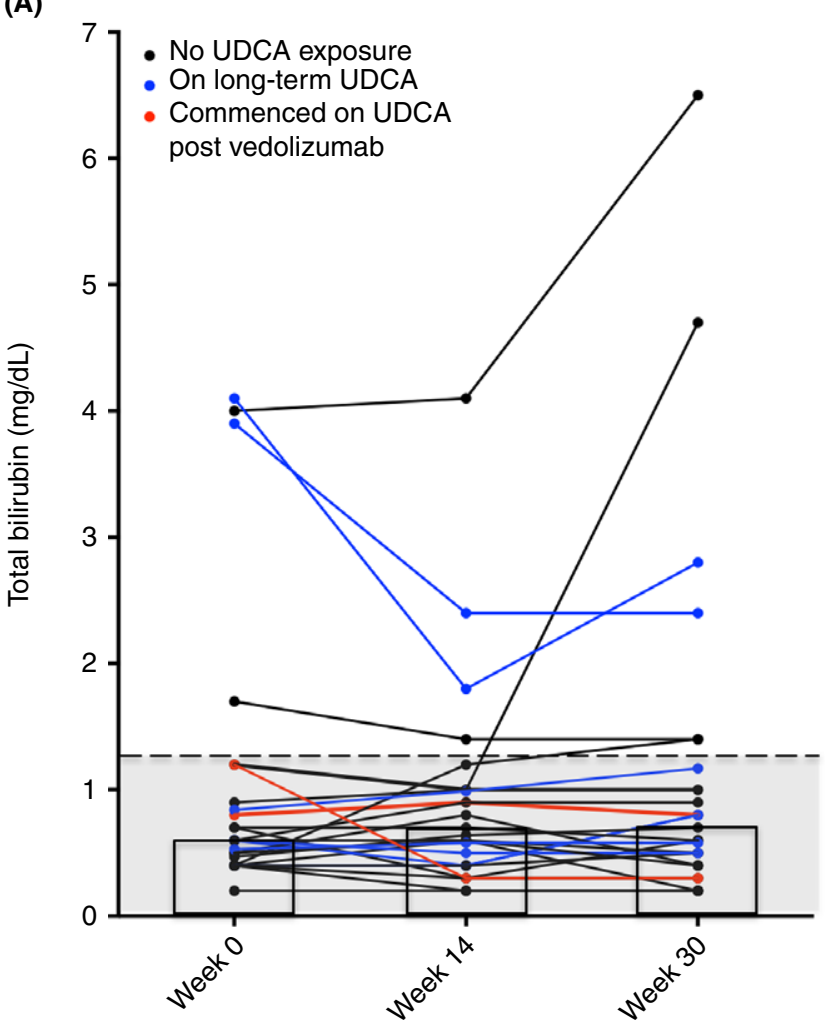

(C)

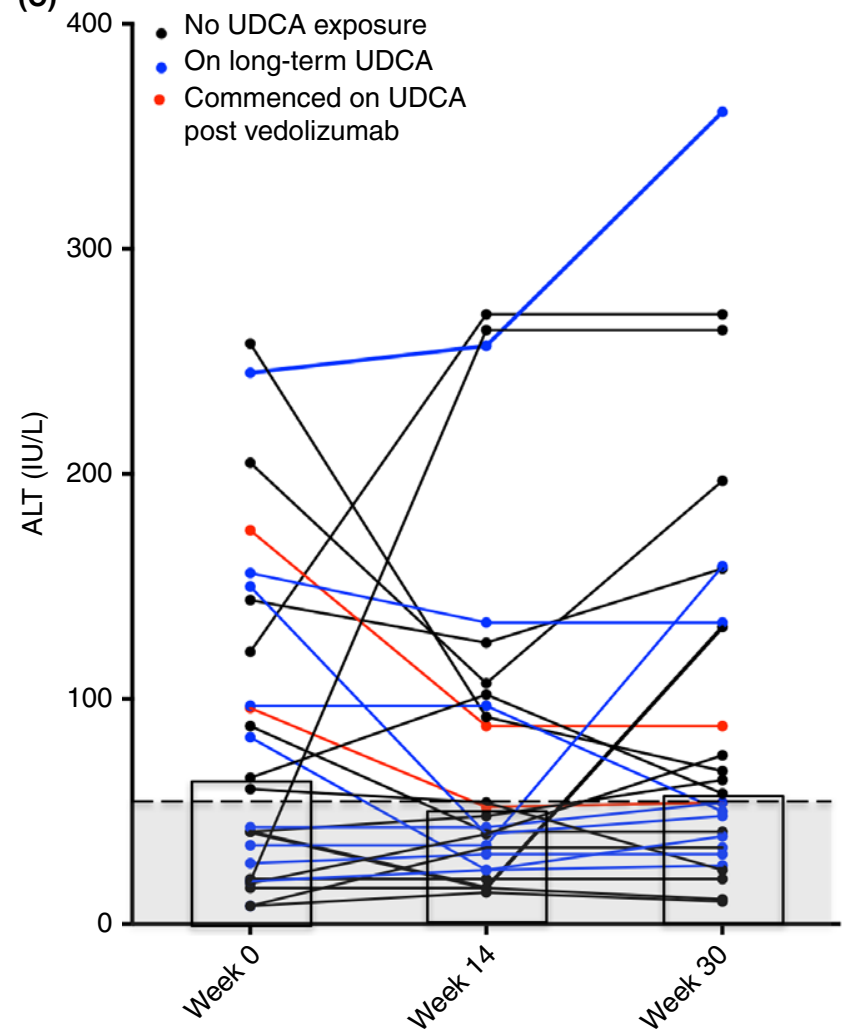

(B)

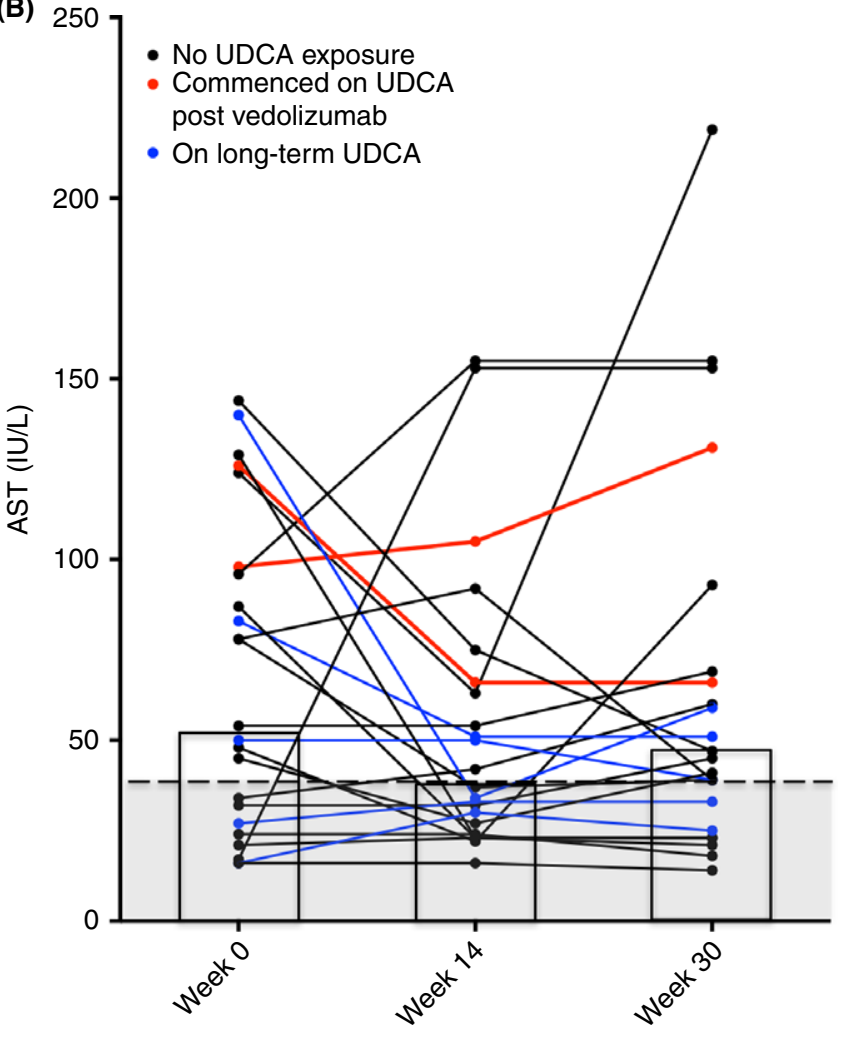

(D)

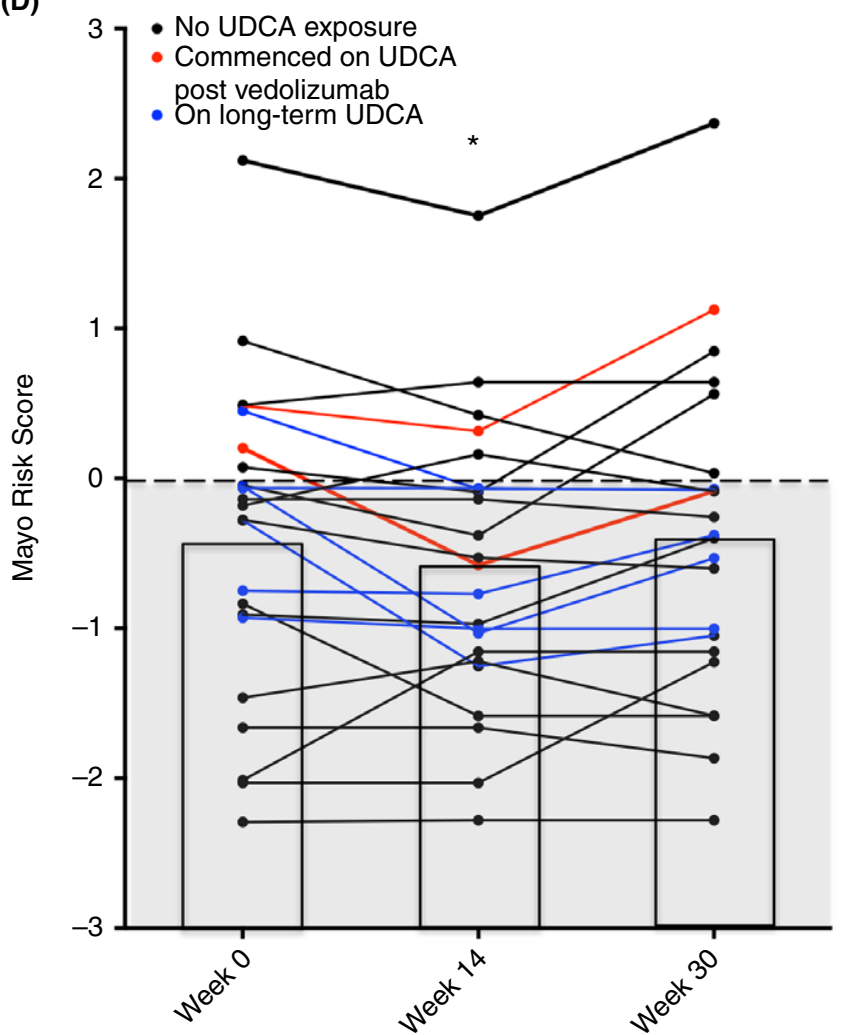

FIG URE 3 Liver biochemistry and Mayo PSC Risk Score before and following 14 and 30 wk' treatment with vedolizumab in patients with IBDPSC. *Indicates significant decrease $(P<0.05)$ from wk 0 level. Shaded region represents normal range of factor. The columns indicate the median value for total bilirubin, AST and ALT and mean value for Mayo PSC Risk Score. (A) Total bilirubin: no change in total bilirubin with treatment. (B) Aspartate aminotransferase (AST) activities; no change with treatment. (C) Alanine aminotransferase (ALT) activities: no change with treatment. (D) Mayo PSC Risk Scores: improvement in Mayo PSC Risk Score from baseline to week $14(P=0.03)$, but not to wk $30(P=0.90)$ 
(A)

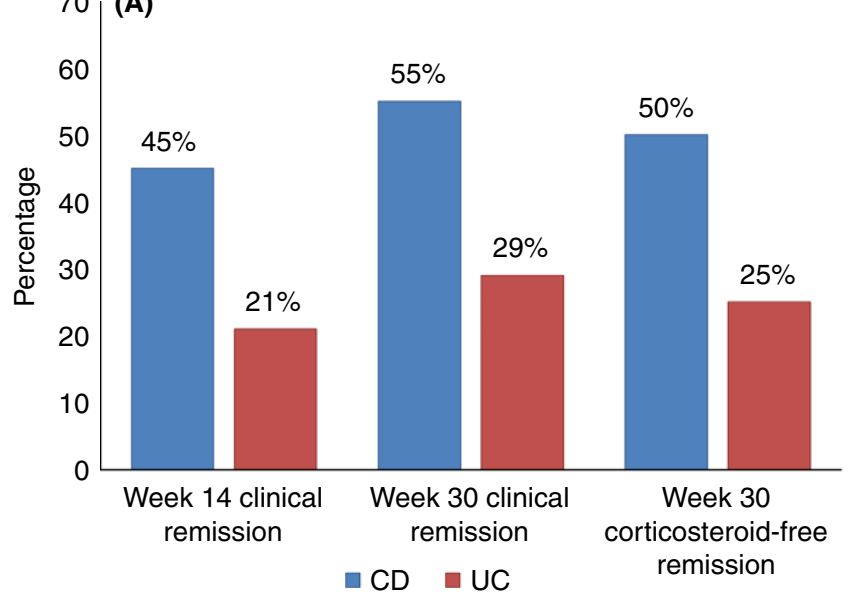

(B)

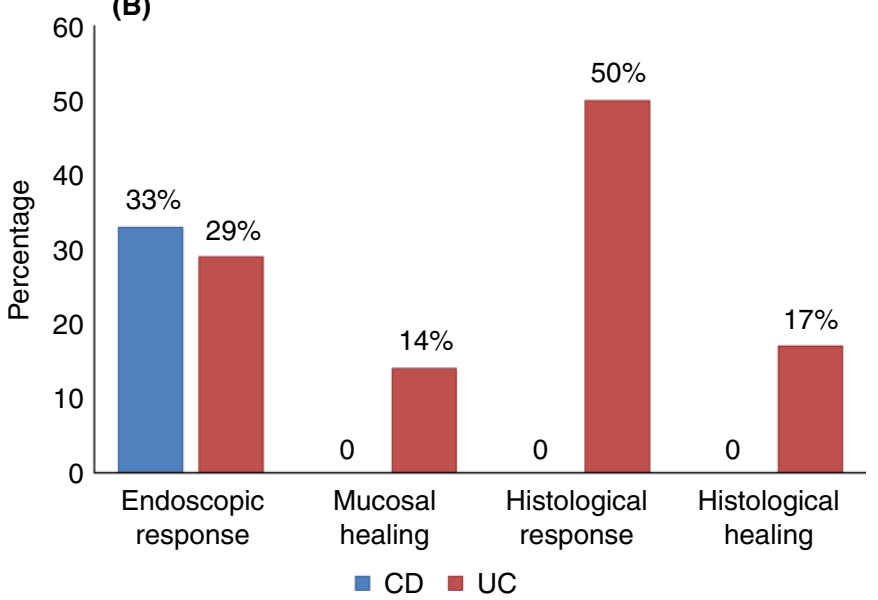

FIGURE 4 Effects of vedolizumab on disease activity in patients with clinically active disease on initiation of therapy. (A) Proportion of patients in clinical remission and corticosteroid-free clinical remission. (B) Proportion of patients with endoscopic response or healing following vedolizumab therapy

the most well described treatment for PSC, indicate that significant improvement in liver biochemistries in patients with decrease serum alkaline phosphatase activities by up to $67 \%^{6,24-26}$ have not been reflected in improved clinical outcomes and in fact, more recently, high-dose ursodeoxycholic acid has been associated with worsening clinical outcomes and the development of colorectal cancer. ${ }^{27,28}$

Whether PSC itself is at all reversible is something that is yet to be determined. In this short-term study, we have relied on improvement in liver biochemistry to determine the utility of vedolizumab in patients with PSC. It is therefore presumed that, in part at least, the damage and increase in alkaline phosphatase in PSC is reversible. This may not be the case and is a limiting factor in all studies examining treatment options for PSC. Currently, trials in PSC therapeutics have been severely hampered by the time taken to reach clinically significant end-points and that there is no well-defined early surrogate marker for disease outcomes. ${ }^{29}$ This study is no different and longer term, multi-centre and case-control studies of patients with PSC and IBD treated with vedolizumab will be required to determine if exposure to vedolizumab alters the rate of development of advanced liver disease, need for liver transplant, colorectal cancer and cholangiocarcinoma despite seeming to have little benefit on liver biochemistry.

In this study, IBD-PSC patients who had active intestinal disease achieved rates of clinical remission with vedolizumab similar to those previously reported. ${ }^{11,30-33}$ However, despite vedolizumab being clinically effective in the IBD-PSC patient cohort, we found low rates of mucosal healing and histological remission. It has previously been reported that vedolizumab achieves mucosal healing in $50 \%$ of UC patients $^{9,31}$ and $20 \%-30 \%$ of CD patients. ${ }^{11,31}$ In our study, no patient with CD and PSC achieved mucosal or histological healing and only one of seven with UC achieved mucosal healing and histological remission. How these rates compare directly to IBD-PSC patients on other therapies is unknown but a recent paper by Krugliak Cleveland et $\mathrm{al}^{34}$ did demonstrate that UC patients with PSC who were in clinical remission were significantly more likely to have endoscopic and histological inflammation compared to UC patients without PSC. This warrants further attention as ongoing histological inflammatory activity ${ }^{35}$ and PSC $^{36}$ are associated with an increased risk of bowel neoplasia. Furthermore, a theoretical concern with the use of vedolizumab is an increased risk of colorectal cancer due to decreased immune surveillance of the gut. Reassuringly, no associated increased risk of colorectal cancer has been found in long-term safety studies on vedolizumab compared to the general IBD population. $^{37}$

Our study has shown that vedolizumab is safely administered to patients with IBD-PSC. In this cohort, seven (21\%) patients ceased vedolizumab therapy after a median of 8 months (IQR: $5.5,8)$ of which six were for primary nonresponse to vedolizumab. One patient had normal liver function tests prior to commencing vedolizumab but developed drug-related hepatotoxicity and was required to cease vedolizumab. Two further patients did develop cholangitis, one of which required liver transplantation for deterioration of liver disease and recurrent cholangitis after 7 months of therapy. The second patient had an elevated liver function profile at baseline that failed to improve after 3 months of vedolizumab therapy and was found to have a dominant biliary stricture on ERCP that required dilation. There were no other severe adverse events associated with vedolizumab use in this population, and the $12 \%$ of patients with minor adverse event were expected and similar as those reported in previous studies. ${ }^{11,30,31}$

There are a number of notable limitations to this study. First, all data collection was performed retrospectively, but, since the included centres are all major referral centres for IBD and liver disease, we were able to collect data obtained from routine clinic visits. Although we strengthened the data quality using objective outcome assessments where possible, there may still be bias present in the clinical follow-up of patients. Secondly, the sample size was small, which may have contributed, for example, to the failure to observe statistical significance in changes in liver biochemistry, particularly at week 30 outcomes where large interquartile ranges are observed. 
However, the absolute difference in the primary outcomes of alkaline phosphatase levels does not appear to be clinically significant even if larger patient numbers were able demonstrates a statistically significant difference. The small sample size, however, also did not allow comparison of liver biochemistry improvement between different sub-groups including those with intra versus extrahepatic PSC or history of liver transplant to be adequately explored. The patients included in this study were also more likely to have CD than UC which is not reflective of the ratios of $C D$ versus $U C$ in the general PSC population. This is likely secondary to the fact that at the time of this study vedolizumab was primarily used to treat the intestinal disease activity rather than the PSC and in some centres, including the University of Chicago, the majority of patients commenced on vedolizumab had $\mathrm{CD} .{ }^{30,38,39,40}$ There is also the possibility that changes in ALP were secondary to other causes like low vitamin D status. Unfortunately, vitamin D levels were not assessed in this study but it is felt the likelihood of this altering the results significantly is low as all patients were treated at large academic centres where Vitamin D levels are routinely assessed and aggressively replaced. Finally, this study is limited by its short duration of followup. Changes in liver biochemistries were only assessed to week 30 of therapy and, therefore, longer term outcomes such as need for liver transplantation, development of cirrhosis or cancer incidence were unable to be assessed. Clearly larger, prospective, multi-centre studies are required to look at this question in more detail.

In conclusion, our study did not demonstrate sustained improvement in liver biochemistries in patients with UC and PSC treated with vedolizumab, and in fact revealed a modest increase in alkaline phosphatase in patients who had normal levels prior to vedolizumab commencement. This increase rarely resulted in discontinuation of vedolizumab, and we have demonstrated that vedolizumab therapy appears safe in patients with PSC, advanced liver disease and a history of orthotopic liver transplantation. In addition, clinical response and remission in IBD activity seems to be similar to the population of patients with IBD without PSC, although rates of mucosal healing may be lower. Future registry studies should focus more on whether vedolizumab can improve long-term clinical outcomes in PSC patients including decreasing the development of new biliary strictures, cirrhosis, need for transplantation and cancer incidence.

\section{ACKNOWLEDGEMENTS}

Declaration of personal interests: Joel Pekow has received grant support from Takeda. David Rubin has received institutional grant support from Abbvie, and consulting or advisory board funding from Verastem, Pfizer and Janssen. Janssen and Takeda and served as a consultant for Abbvie, Janssen, Takeda, Amgen, Pfizer and UCB. Peter R. Gibson has served as consultant or advisory board member for AbbVie, Ferring, Janssen, Merck, Nestle Health Science, Danone, Allergan, Celgene and Takeda. His institution has received speaking honoraria from AbbVie, Janssen, Ferring, Takeda, Fresenius Kabi, Mylan and Pfizer. He has received research grants for investigatordriven studies from AbbVie, Janssen, Falk Pharma, Danone and A2
Milk Company. Britt Christensen has received grants from Takeda and Pfizer. There are no other expressed conflicts of interest with respect to the submitted work. Writing assistance: No support provided.

Declaration of funding interests: This work was supported by the $\mathrm{NIH}$ [grant numbers P30DK42086, K08DK090152 to JP].

\section{AUTHORSHIP}

Guarantor of article: Britt Christensen.

Author contributions: B. Christensen contributed to conception and design, acquisition, analysis or interpretation, drafting of manuscript and approved final version to be submitted. D. Micic contributed to conception and design, acquisition, analysis or interpretation, drafting of manuscript and approved final version to be submitted. P. R. Gibson contributed to conception and design, acquisition, analysis or interpretation, drafting of manuscript and approved final version to be submitted. A. Yarur contributed to conception and design, acquisition, analysis or interpretation, drafting of manuscript and approved final version to be submitted. E. Bellaguarda contributed to acquisition, analysis or interpretation, critical review of manuscript and approved final version to be submitted. P. Corsello contributed to acquisition, analysis or interpretation, critical review of manuscript and approved final version to be submitted. J. N. Gaetano contributed to acquisition, analysis or interpretation, critical review of manuscript and approved final version to be submitted. J. Kinnucan contributed to acquisition, analysis or interpretation, critical review of manuscript and approved final version to be submitted. V. L. Rao contributed to acquisition, analysis or interpretation, critical review of manuscript and approved final version to be submitted. S. Reddy contributed to acquisition, analysis or interpretation, critical review of manuscript and approved final version to be submitted. S. Singh contributed to acquisition, analysis or interpretation, critical review of manuscript and approved final version to be submitted. D. T. Rubin contributed to conception and design and critical review of manuscript and approved final version to be submitted. J. Pekow contributed to conception and design, acquisition, analysis or interpretation, drafting of manuscript and approved final version to be submitted. All authors approved the final version of the manuscript.

\section{ORCID}

B. Christensen (iD) http://orcid.org/0000-0002-8746-4275

\section{REFERENCES}

1. Hirschfield GM, Karlsen TH, Lindor KD, et al. Primary sclerosing cholangitis. Lancet. 2013;382:1587-1599.

2. Boonstra K, van Erpecum KJ, van Nieuwkerk KM, et al. Primary sclerosing cholangitis is associated with a distinct phenotype of inflammatory bowel disease. Inflamm Bowel Dis. 2012;18:2270-2276.

3. Eaton JE, Talwalkar JA, Lazaridis KN, et al. Pathogenesis of primary sclerosing cholangitis and advances in diagnosis and management. Gastroenterology. 2013;145:521-536. 
4. Porayko MK, Wiesner RH, LaRusso NF, et al. Patients with asymptomatic primary sclerosing cholangitis frequently have progressive disease. Gastroenterology. 1990;98:1594-1602.

5. Paumgartner G, Beuers U. Ursodeoxycholic acid in cholestatic liver disease: mechanisms of action and therapeutic use revisited. Hepatology. 2002;36:525-531.

6. Lindor KD. Ursodiol for primary sclerosing cholangitis. Mayo Primary Sclerosing Cholangitis-Ursodeoxycholic Acid Study Group. N Engl J Med. 1997;336:691-695.

7. Lindor KD, Kowdley KV, Luketic VA, et al. High-dose ursodeoxycholic acid for the treatment of primary sclerosing cholangitis. Hepatology. 2009;50:808-814.

8. Lobaton T, Vermeire S, van Assche G, et al. Review article: antiadhesion therapies for inflammatory bowel disease. Aliment Pharmacol Ther. 2014;39:579-594.

9. Feagan BG, Rutgeerts P, Sands BE, et al. Vedolizumab as induction and maintenance therapy for ulcerative colitis. $N$ Engl J Med. 2013;369:699-710.

10. Sandborn WJ, Feagan BG, Rutgeerts P, et al. Vedolizumab as induction and maintenance therapy for Crohn's disease. N Engl J Med. 2013;369:711-721.

11. Dulai PS, Singh S, Jiang $X$, et al. The real-world effectiveness and safety of vedolizumab for moderate-severe Crohn's disease: results from the US VICTORY Consortium. Am J Gastroenterol. 2016;111:1147-1155.

12. Eksteen B, Grant AJ, Miles A, et al. Hepatic endothelial CCL25 mediates the recruitment of CCR9+ gut-homing lymphocytes to the liver in primary sclerosing cholangitis. J Exp Med. 2004;200:1511-1517.

13. Hillan KJ, Hagler KE, MacSween RN, et al. Expression of the mucosal vascular addressin, MAdCAM-1, in inflammatory liver disease. Liver. 1999;19:509-518.

14. Lim TY, Pavlidis P, Gulati S, et al. Vedolizumab in inflammatory bowel disease associated with autoimmune liver disease pre- and postliver transplantation: a case series. Inflamm Bowel Dis. 2016;22: E39-E40.

15. Damas OM, Estes DE, Pena Polanco NA, et al. Sa1580 treatment of inflammatory bowel disease with vedolizumab for patients with coexisting primary sclerosing cholangitis: report of a nested casecontrol study. Gastroenterology. 2016;150:S1074.

16. Satsangi J, Silverberg MS, Vermeire S, et al. The Montreal classification of inflammatory bowel disease: controversies, consensus, and implications. Gut. 2006;55:749-753.

17. Dickson ER, Grambsch PM, Fleming TR, et al. Prognosis in primary biliary cirrhosis: model for decision making. Hepatology. 1989;10:1-7.

18. Harvey RF, Bradshaw JM. A simple index of Crohn's-disease activity. Lancet. 1980;1:514.

19. Walmsley RS, Ayres RC, Pounder RE, et al. A simple clinical colitis activity index. Gut. 1998;43:29-32.

20. Daperno M, D'Haens G, Van Assche G, et al. Development and validation of a new, simplified endoscopic activity score for Crohn's disease: the SES-CD. Gastrointest Endosc. 2004;60:505-512.

21. Schroeder KW, Tremaine WJ, Ilstrup DM. Coated oral 5-aminosalicylic acid therapy for mildly to moderately active ulcerative colitis. A randomized study. N Engl J Med. 1987;317:1625-1629.

22. Joseph N, Weber C. Pathology of Inflammatory Bowel Disease. In: Baumgart D, ed. Crohn's Disease \& Ulcerative Colitis. New York: Springer; 2011:287-306.

23. Rupp C, Rossler A, Halibasic E, et al. Reduction in alkaline phosphatase is associated with longer survival in primary sclerosing cholangitis, independent of dominant stenosis. Aliment Pharmacol Ther. 2014;40:1292-1301.

24. Beuers U, Spengler U, Kruis W, et al. Ursodeoxycholic acid for treatment of primary sclerosing cholangitis: a placebo-controlled trial. Hepatology. 1992;16:707-714.
25. Mitchell SA, Bansi DS, Hunt N, et al. A preliminary trial of high-dose ursodeoxycholic acid in primary sclerosing cholangitis. Gastroenterology. 2001;121:900-907.

26. Olsson R, Boberg KM, de Muckadell OS, et al. High-dose ursodeoxycholic acid in primary sclerosing cholangitis: a 5-year multicenter, randomized, controlled study. Gastroenterology. 2005;129:1464-1472.

27. Eaton JE, Silveira MG, Pardi DS, et al. High-dose ursodeoxycholic acid is associated with the development of colorectal neoplasia in patients with ulcerative colitis and primary sclerosing cholangitis. Am J Gastroenterol. 2011;106:1638-1645.

28. Imam MH, Sinakos E, Gossard AA, et al. High-dose ursodeoxycholic acid increases risk of adverse outcomes in patients with early stage primary sclerosing cholangitis. Aliment Pharmacol Ther. 2011;34:1185-1192.

29. Goode EC, Rushbrook SM. A review of the medical treatment of primary sclerosing cholangitis in the 21st century. Ther Adv Chronic Dis. 2016;7:68-85.

30. Amiot A, Grimaud JC, Peyrin-Biroulet L, et al. Effectiveness and safety of vedolizumab induction therapy for patients with inflammatory bowel disease. Clin Gastroenterol Hepatol. 2016;14:e2.

31. Vivio EE, Kanuri N, Gilbertsen JJ, et al. Vedolizumab effectiveness and safety over the first year of use in an IBD clinical practice. $J$ Crohns Colitis. 2015;10:402-409.

32. Baumgart DC, Bokemeyer B, Drabik A, et al. Vedolizumab induction therapy for inflammatory bowel disease in clinical practice-a nationwide consecutive German cohort study. Aliment Pharmacol Ther. 2016;43:1090-1102.

33. Stallmach A, Langbein C, Atreya R, et al. Vedolizumab provides clinical benefit over 1 year in patients with active inflammatory bowel disease - a prospective multicenter observational study. Aliment Pharmacol Ther. 2016;44:1199-1212.

34. Cleveland NK, Rubin DT, Hart J, et al. 'Patients with ulcerative colitis and primary sclerosing cholangitis frequently have subclinical inflammation in the proximal colon'. Clin Gastroenterol Hepatol. 2017; in press.

35. Rutter M, Saunders B, Wilkinson K, et al. Severity of inflammation is a risk factor for colorectal neoplasia in ulcerative colitis. Gastroenterology. 2004;126:451-459.

36. Kornfeld D, Ekbom A, Ihre T. Is there an excess risk for colorectal cancer in patients with ulcerative colitis and concomitant primary sclerosing cholangitis? A population based study. Gut. 1997;41:522-525.

37. Colombel JF, Sands BE, Rutgeerts $P$, et al. The safety of vedolizumab for ulcerative colitis and Crohn's disease. Gut. 2016;66:839-851.

38. Kopylov U, Ron Y, Avni-Biron I, et al. Efficacy and safety of vedolizumab for induction of remission in inflammatory bowel disease-the Israeli real-world experience. Inflamm Bowel Dis. 2017;23:404-408.

39. Shelton E, Allegretti JR, Stevens B, et al. Efficacy of vedolizumab as induction therapy in refractory IBD patients: a multicenter cohort. Inflamm Bowel Dis. 2015;21:2879-2885.

40. Christensen B, Goeppinger SR, Colman RJ, et al. Tu1350 vedolizumab in the treatment of IBD: the University of Chicago experience. Gastroenterology. 2015;148:S-866.

How to cite this article: Christensen B, Micic D, Gibson PR, et al. Vedolizumab in patients with concurrent primary sclerosing cholangitis and inflammatory bowel disease does not improve liver biochemistry but is safe and effective for the bowel disease. Aliment Pharmacol Ther. 2018;47:753-762. https://doi.org/10.1111/apt.14525 